\title{
New Therapeutic Approaches for Airway Hyperimmune Response are Required
}

\author{
Pramod Upadhyay \\ Scientist, National Institute of Immunology, Aruna Asaf Ali Marg, New Delhi 110067, India. \\ pkumar@nii.ac.in
}

I ndia is among the most densely populated countries of the world and it is undergoing rapid urbanization and economic changes and simultaneously the prevalence of asthma and allergies are increasing. Similar trends have been observed in other developing countries. In this context the study by Raj, et al. [1] on aeroallergen sensitization in childhood asthmatics in northern India is significantly important. In this study, the skin prick test (SPT) was performed on 180 children and very exciting results were obtained; close to $44 \%$ of the children were negative for atopy and in remaining 56\% interesting pattern of sensitization was observed which appears to be unique to north India. This paper reestablishes the utility of the SPT.

This paper can stimulate immunologists in this part of the world because not much understanding has been achieved in the mechanism which leads to aeroallergen provoked hyper-immune response. It was not long ago that dendritic cells (DCs) were shown as key pro-inflammatory cells that are responsible for $\mathrm{Th} 2$ cell stimulation during airway inflammation [2]. Subsequently, it was shown that the aeroallergen provocation induces the rapid accumulation of CD11c+MHC class II DCs in the lungs and this process is driven by DCs precursors from blood [3]. This Th2 cell-driven inflammation is believed to generate an abnormal response to airborne particles. These reactions are normally suppressed by $\mathrm{T}$ - regulatory cells and the anti-inflammatory cytokine IL-10 is suggested to play a central role. It is generally believed that in asthmatic individuals there is a breakdown in these regulatory mechanisms [4]. Interestingly, the airways hyperresponsiveness susceptibility is inversely related to aeroallergen exposure level and the high exposure confers protection and it is the compromised antigen surveillance by airway mucosal DCs which results in defective functional programming of T-regulatory cells ultimately generating the hyper-responsiveness [5]. Though these findings are providing some insight into the airways hyper immune-response in laboratory animals but these are far from therapeutic approaches.
In a detailed investigation, a confirmation of "biodiversity hypothesis," has been made [6]. In this study it was shown that the atopic individuals had significantly lower generic diversity of gammaproteobacteria on their skin and lower environmental biodiversity of their homes compared with healthy individuals. It is a serious concern whether the climate change will one day modify the entire environment and produce high and similar prevalence of airways hyper-responsiveness everywhere. It is not the innate and adaptive immune responses alone which lead to the airways hyper immune-response, the skin microbiota also functions in educating the immune system. The skin bacteria are highly diverse and variable. An understanding of the ability of skin microbiome to modulate the immune response possibly can lead to novel pro and anti-microbial therapeutic approaches for managing the airways hyper immune response.

Competing interests: None stated; Funding: Nil.

\section{REFERENCES}

1. Raj D, Lodha R, Pandey A, Mukherjee A, Agrawal A, Kabra SK. Aeroallergen sensitization in childhood asthmatics in Northern India. Indian Pediatr. 2013;50:1113-8.

2. van Rijt LS, Jung S, Kleinjan A, Vos N, Willart M, Duez C, et al. In vivo depletion of lung $\mathrm{CD} 11 \mathrm{c}+$ dendritic cells during allergen challenge abrogates the characteristic features of asthma. J Exp Med. 2005;201:981-91.

3. Veres TZ, Voedisch S, Spies E, Valtonen J, Prenzler F, Braun A. Aeroallergen challenge promotes dendritic cell proliferation in the airways. J Immunol. 2013;190:897-903.

4. Lloyd CM, Hawrylowicz CM. Regulatory $\mathrm{T}$ cells in asthma. Immunity. 2009;31:438-49.

5. Strickland DH, Thomas JA, Mok D, Blank F, McKenna $\mathrm{KL}$, Larcombe AN, et al. Defective aeroallergen surveillance by airway mucosal dendritic cells as a determinant of risk for persistent airways hyperresponsiveness in experimental asthma. Mucosal Immunol. 2012;5:332-41.

6. Hanski I, von HL, Fyhrquist N, Koskinen K, Torppa K, Laatikainen T, et al. Environmental biodiversity, human microbiota, and allergy are interrelated. Proc Natl Acad Sci USA. 2012;109:8334-9. 